\title{
The added value of the EQ-5D with a cognition dimension in injury patients with and without traumatic brain injury
}

\author{
A. J. L. M. Geraerds ${ }^{1}$ (D) Gouke J. Bonsel ${ }^{1,3} \cdot$ Mathieu F. Janssen $^{2} \cdot$ M. A. de Jongh ${ }^{4} \cdot$ Inge Spronk $^{1} \cdot$ Suzanne Polinder $^{1}$. \\ Juanita A. Haagsma ${ }^{1}$
}

Accepted: 18 February 2019 / Published online: 28 February 2019

(c) The Author(s) 2019

\begin{abstract}
Purpose This study investigated the psychometric gain, if any, from the extension of the EQ-5D with a cognition bolt-on $(\mathrm{EQ}-5 \mathrm{D}+\mathrm{C})$ in a large cohort injury patients with and without traumatic brain injury (TBI).

Methods Hospitalized adult injury patients filled out a survey 1 month after initial admission. The survey included the EQ5D-3L, the cognition bolt-on item in EQ-5D format, and the visual analogue scale (EQ-VAS). We compared ceiling and other distributional effects between EQ-5D and EQ-5D + C and TBI and non-TBI group, and assessed convergent validity using the predictive association with EQ-VAS. Also, we assessed explanatory power using regression analysis, and classification efficiency using Shannon indices.

Results In total, 715 TBI patients and 1978 non-TBI patients filled out the EQ-5D + C and EQ-VAS. Perfect health was reported by $7.9 \%(N=214)$ on the EQ-5D, and $7.3 \%(N=197)$ on the EQ-5D + C. Convergent validity was highest for EQ-5D $+C$ in the TBI group (Spearman's rank correlation coefficient $=-0.736$ ) and lowest for EQ-5D in the non-TBI group (Spearman's rank correlation coefficient $=-0.652$ ). For both TBI and non-TBI groups, the explanatory power of EQ-5D $+\mathrm{C}$ was slightly higher than of EQ-5D $\left(R^{2}=0.56\right.$ vs. 0.53 for TBI; $R^{2}=0.47$ vs. 0.45 for non-TBI). Absolute classification efficiency was higher for EQ-5D $+C$ than for EQ-5D in both TBI groups, whereas relative classification efficiency was similar. Conclusions Psychometric performance in general of both the EQ-5D and EQ-5D + C was better in TBI patients. Adding a cognitive bolt-on slightly improved the psychometric performance of the EQ-5D-3L.
\end{abstract}

Keywords HRQoL $\cdot$ EQ-5D · Cognition · TBI

\section{Background}

Currently, the measurement of health-related quality of life (HRQoL) is standard practice in evaluating the impact of health interventions [1]. HRQoL instruments can be categorized as generic and disease-specific measures, where a

A. J. L. M. Geraerds

a.geraerds@erasmusmc.nl

1 Department of Public Health, Erasmus MC, University Medical Center Rotterdam, P.O. Box 2040, 3000 CA Rotterdam, The Netherlands

2 Section Medical Psychology and Psychotherapy, Department of Psychiatry, Erasmus MC, Rotterdam, The Netherlands

3 Division Mother and Child, Utrecht University Medical Center, Utrecht, The Netherlands

4 Department Trauma TopCare, ETZ Hospital, Hilvarenbeekseweg 60, 5022 GC Tilburg, The Netherlands particular subclass of instruments are preference-based or 'utility' measures, which can be used in economic evaluations [2].

One of the generic instruments that is widely implemented is the EQ-5D. The EQ-5D is a self-assessment instrument that consists of five items on the following dimensions: mobility, self-care, usual activities, pain/discomfort, and anxiety/ depression and a visual analogue scale (VAS) [3]. The five dimensions can be scored using 3- or 5-level ordinal response options. One major advantage of the EQ-5D over other generic HRQoL measurement instruments is its brevity and subsequent low burden to fill out $[4,5]$. However, a downside of the brevity may be that important information for HRQoL is not included in the EQ-5D dimensions, resulting in an instrument that may be unable to capture certain health effects and that is not sensitive for the measurement of HRQoL of all conditions [6, 7]. Moreover, the EQ-5D is known to measure mainly physical dimensions of health, and lacking information 
on social domains and sensitivity on psychological domains. Therefore, the EQ-5D is inconsistent in some populations [8]. A solution to increase coverage of the EQ-5D may be to add a dimension (a 'bolt-on') covering a specific health problem or dysfunction relevant to any particular condition or disease [9].

Over time, different bolt-on items to enrich the EQ-5D have been suggested. One of these bolt-on items is cognition [10]. Cognition can be operationalized with attributes like concentration, memory, intellectual competence, and coherence [10]. A cognition bolt-on can be motivated from theoretical reasons or from the pragmatic observation that cognition affecting diseases and conditions are common (dementia, Parkinson's disease, injury, birth trauma, and congenital neurological conditions).

Even though many studies already use the cognition bolton in the measurement of HRQoL with the EQ-5D, limited evidence on the added value of the cognitive bolt-on in patient groups with cognitive impairments exists. Wolfs et al. [11] found that in a population of elderly patients with cognitive impairments, the addition of a cognitive dimension had no effect on the construct validity. Ophuis et al. (Working paper: Health-related quality of life in injury patients: the added value of extending the EQ-5D-3L with a cognitive dimension) found that adding a cognition dimension to the EQ-5D in a heterogeneous sample of injury patients, including patients with mostly mild traumatic brain injury (TBI), had a similar impact on EQ-VAS scores as any of the existing five EQ-5D dimensions. However, both studies were lacking a clear differentiation between the effects of adding a cognitive dimension to the EQ-5D in patients with and without suspected cognitive impairments. Therefore, this study can provide a more accurate insight in the additional value of a cognition dimension in the EQ-5D for TBI patients.

This study investigated the added value of a cognition dimension to the EQ-5D in a population of injury patients. The added value was analyzed in the form of the distributional benefit, the convergent validity, explanatory power, and classification efficiency of the EQ-5D and EQ-5D $+C$ in a large sample of TBI and non-TBI patients who were admitted to the hospital due to their injury. We explored this by comparing ceiling and other distribution effects and convergent validity using the predictive association with the EQ-VAS. Also, we assessed the explanatory power using regression analyses, and classification efficiency using Shannon indices. Each of these aspects were determined for patients with and without TBI.

\section{Methods}

\section{Research population and data collection}

The Brabant Injury Outcome Surveillance (BIOS) study is a prospective cohort follow-up study of adult injury patients
(18 years or older) who were admitted to one of the 10 participating hospitals through the emergency department (ED), and who survived until hospital discharge [12]. The inclusion of the study was conducted from 1 August 2015 until 30 November 2016 . The exclusion criteria were the inability to understand the Dutch language, the absence of a permanent address of residence, and the suspected presence of a pathological fracture due to a malignancy or metastasis [12]. The study was approved by the Medical Ethics Committee of the Province of Brabant (METC code: NL50258.028.14).

The information on age, gender, nature, and severity of the injury were derived from the hospital registries. The severity of the injury was defined by the Injury Severity Score (ISS) [13]. The ISS is based on the square of the highest Abbreviated Injury Scales (AIS) of the three most severely injured body regions. The AIS describes the type, location, and severity of an injury [14]. Patients with an ISS of 16 and more are defined as severely injured. With the AIS score, it can be determined whether a patient has TBI, and what the severity level of the TBI is (mild, moderate, or severe). TBI was defined as mild with AIS head $<3$, moderate with AIS head $=3$, and severe with an AIS head $>3$ [12]. Additional data were collected by a postal survey that patients received 1 month after injury [12]. The survey included the EQ-5D +C, the EQ-VAS, the highest level of education, and the existence of comorbidity.

\section{HRQoL data}

The EQ-5D consists of five items on different dimensions of health (one item per dimension): mobility, self-care, usual activity, pain/discomfort, and anxiety/depression. The questions on the different dimensions are available in two versions: a three-level version and a five-level version. We used the three-level version (EQ-5D-3L). In the $3 \mathrm{~L}$ version, the answer options to each question are 'no problems,' 'some problems,' and 'extreme problems'/'unable to perform.' Data can be represented by means of a profile summarizing the respondents' reported health problems defining the severity level (where 1 means no problems), e.g., '21332,' and used in a descriptive way and, after summarizing response into an unweighted summary score, also referred to as 'misery index' (range $0-15$ ) or a so-called 0-1 utility score. Furthermore, the respondents were asked to rate their health from 0 to 100 on a VAS scale, where 0 is the worst imaginable health state, and 100 is the best imaginable health state. The score that is provided in this question by the respondent is the EQ-VAS score.

In the BIOS study, an additional dimension was added to the EQ-5D questionnaire for cognition. This bolt-on should capture information on cognitive functioning, operationalized as concentration, memory, and IQ [10]. The cognition bolt-on consisted of one question, and was framed like 
the other dimension questions, with the same number of response options [3]. The instruction was (translated from the Dutch questionnaire): By placing a check mark in one box in each group below, please indicate which statement best describes your own state of health. The cognition bolton item was worded as follows: cognition (such as memory, concentration). The answer options were: I have no problems with my cognitive functioning; I have some problems with my cognitive functioning; I have severe problems with my cognitive functioning.

\section{Data analysis}

The data analyses were performed with SPSS version 24 . The responses of the patients to the question on the highest level of education were categorized in a variable with the values low, medium, and high education level. Comorbidity status was determined per patient as the number of pre-existing conditions. Respondents were included in the analyses if all questions of the EQ-5D including the cognition dimension and the EQ-VAS score were answered. The frequencies of the socio-demographics were determined with frequency analyses in SPSS. Furthermore, independent sample $t$ tests were performed on the frequencies of the socio-demographics, comparing the group of TBI patients with the group of non-TBI patients. To determine whether there was a distributional effect in terms of ceiling effect, the proportion of perfect health profiles (11111 for EQ-5D and 111111 for EQ-5D+C) among all observed profiles was determined (the higher the share, the more ceiling).

The convergent validity of the EQ-5D and the EQ-5D + C was measured by determining the association between the EQ-VAS, the EQ-5D, and the EQ-5D + C, respectively. We first calculated the misery index for both the EQ-5D and the EQ-5D $+C$, consisting of the sum of the levels of the five dimensions (e.g., health profile 11111 had a misery index of 5), where the highest score 15 represents the poorest health. Next, Spearman's rank correlation coefficients between the EQ-5D and EQ-5D + C misery indices and EQ-VAS were determined for TBI and non-TBI patients, after it was confirmed that the assumptions of the Spearman's rank correlation were met [15].

In order to determine the explanatory power of EQ-5D and EQ-5D $+C$, we performed univariate and multivariable analyses using the EQ-VAS as dependent variable. With the univariate analyses we tested whether all dimensions of the EQ-5D $(+C)$ were related to the EQ-VAS. The levels 'some problems' and 'extreme problems' of all dimensions, including the cognitive dimension, were used to predict the EQ-VAS. The two severity levels were recoded into dummy variables, with the 'no problems' level as the reference category. With each of the dummy variables, the EQ-VAS score was then predicted. Thereafter, multivariable analyses were done, as the assumptions of the linear regression model were met, with different combinations of the dimensions of the EQ-5D and the cognitive dimension in the model. The combinations consisted of the original EQ-5D dimensions, the EQ-5D dimensions with the cognitive dimension, and all combinations of five out of the six dimensions. An additional model was tested for the EQ-5D and the EQ-5D + C for both groups with the backward deletion strategy.

To determine the classification efficiency of both the EQ-5D and the EQ-5D + C, the Shannon index (H') and the Shannon Evenness index (J') were determined [16]. These two indices provide information on the ability of the EQ-5D $(+C)$ to measure diversity in the population [17]. The Shannon index was calculated with the following formula: $H^{\prime}=-\sum_{i=1}^{c} p_{\mathrm{i}}{ }^{2} \log p_{\mathrm{i}}$, where $\mathrm{p}_{\mathrm{i}}$ is the proportion of people with a certain health profile, and $C$ is the total number of possible health profiles. The higher the value of $H^{\prime}$, the more information is captured by the EQ-5D or EQ-5D $+\mathrm{C}$. The total number of possible health profiles is $3 * 3 * 3 * 3 * 3=243$ for the EQ-5D, and 729 for the EQ-5D + C. The Shannon Evenness index was calculated with the formula: $J^{\prime}=$ $H^{\prime} / H_{\text {max }}^{\prime}$, where $H_{\text {max }}^{\prime}$ is ${ }^{2} \log C$ and indicates the total number of possible health profiles. The Shannon Evenness index increases if the extra dimension is used to make more distinction between patients and flattens the distribution into more different health profiles [18]. According to Pielou [19], any assessment of $H^{\prime}$ using a sample of the total 'true' population will lead to an underestimation of information captured, with a magnitude of $(C-1) / 2 N$. This underestimation may be considerable when $C$ is large such as in the EQ-5D, but especially EQ-5D $+C$. Therefore, we also calculated adjusted values of $H^{\prime}$ and $J^{\prime}$, taking this underestimation bias into account.

\section{Hypotheses}

The following five hypotheses were formulated:

- The ceiling with the EQ-5D $+\mathrm{C}$ is less than with the EQ-5D;

- The convergent validity of the EQ-5D + C with the EQVAS is higher than the convergent validity of the EQ-5D with the EQ-VAS in the group of TBI patients;

- The explanatory power of the EQ-5D + C is higher compared to the EQ-5D in TBI patients due to specific cognitive symptoms after TBI;

- The explanatory power of the EQ-5D is higher in nonTBI patients than in TBI patients due to more heterogeneity in type and nature of injury;

- Absolute classification efficiency of the EQ-5D + C is higher compared to the EQ-5D in the group of TBI patients, while relative classification efficiency is similar. 


\section{Results}

\section{Study population}

The flow chart of the selection of the study population can be found in Fig. 1. In total, 9774 people received a questionnaire 1 month after injury. Out of these 9774 people, $2693(27.6 \%)$ filled out each item of the EQ-5D + C and EQ-VAS. Approximately, one in four was diagnosed with TBI $(n=715 ; 26.6 \%)$, where the majority of the TBI patients suffered from mild TBI $(n=550 ; 76.9 \%)$. The socio-demographics of the respondents are summarized in Table 1. The proportion of males was significantly higher for the group of TBI respondents $(57.3 \%)$ than for the group of nonTBI respondents $(48.4 \%)(p<0.05)$. TBI patients reported problems most frequently on the pain dimension (70.1\%), whereas the non-TBI patients reported problems most frequently on the usual activities dimension (87.3\%). The average ISS score was significantly higher for the group of nonTBI respondents than for the group of TBI respondents $(6.83$ vs. $6.05, p<0.05)$.

\section{Frequency of unique health states and ceiling effects}

Of the 2693 respondents, 214 people (7.9\%) reported a perfect health state on the EQ-5D, and 197 (7.3\%) on the EQ-5D + C. In the group of TBI patients, there were 129 (18.0\%) with perfect health on the EQ-5D, and 117 (16.4\%) with perfect health on the EQ-5D+C. In the non-TBI group, the numbers were $85(4.3 \%)$ for the EQ-5D, and $80(4.0 \%)$ for the EQ-5D + C.

\section{Convergent validity}

Table 2 shows that convergent validity was highest for TBI group (EQ-5D + C: Spearman's rank correlation coefficient $=-0.736$; EQ-5D: Spearman's rank correlation coefficient $=-0.719)$ and lowest in the non-TBI group $(\mathrm{EQ}-5 \mathrm{D}+\mathrm{C}$ : Spearman's rank correlation coefficient $=-0.665$; EQ-5D: Spearman's rank correlation coefficient $=-0.652$ ). Note that health increases with a lower score in EQ-5D misery index and a higher score in EQ-VAS.

\section{Explanatory power}

The univariate regression analyses of the EQ-5D + C dimensions showed that all dimensions were significantly associated with the EQ-VAS score in both the group of TBI patients and the group of non-TBI patients (Appendix Table 5). The observed directions of the dimension effects were in all cases as expected (more problems, negative coefficient), except for the level 2 effect of usual activities in the non-TBI group. Also, the magnitude of the effects (level 3 larger than level 2) generally conformed to the expectations. The explained variance of the EQ-VAS was in the TBI group highest for usual activity level 3 (24.3\%). In the non-TBI group, the explained variance of the EQ-VAS was highest for self-care level 3 and usual activities level 3 (both

Fig. 1 Patient flow chart

\section{Research population $n=9774$}

\section{Filled out EQ-5D+C questionnaire after 1 month $n=2693$}

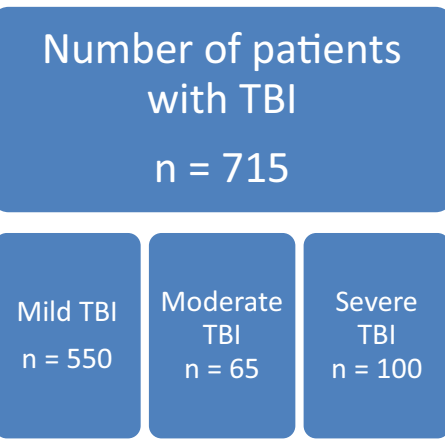

Number of patients with no TBI $n=1978$ 
Table 1 Characteristics of research population

\begin{tabular}{|c|c|c|c|c|}
\hline Demographics of research population & Research population & TBI & Non-TBI & $p$ value \\
\hline$n$ & 2693 & 715 & 1978 & \\
\hline Mean age (SD) & $64.0(18.3)$ & $61.4(18.6)$ & $65.0(18.1)$ & $<0.001^{*}$ \\
\hline Females & $1326(49.2 \%)$ & $305(42.7 \%)$ & $1021(51.6 \%)$ & $<0.001^{*}$ \\
\hline \multicolumn{5}{|l|}{ Education level } \\
\hline Low & $898(33.3 \%)^{\mathrm{a}}$ & $233(32.6 \%)^{\mathrm{b}}$ & $665(33.6 \%)^{\mathrm{c}}$ & 0.616 \\
\hline Medium & $963(35.8 \%)^{\mathrm{a}}$ & $258(36.1 \%)^{\mathrm{b}}$ & $705(35.6 \%)^{\mathrm{c}}$ & 0.833 \\
\hline High & $752(27.9 \%)^{\mathrm{a}}$ & $198(27.7 \%)^{\mathrm{b}}$ & $554(28.0 \%)^{\mathrm{c}}$ & 0.872 \\
\hline \multicolumn{5}{|l|}{ ISS scores } \\
\hline $1-3$ & $609(22.6 \%)^{\mathrm{d}}$ & $358(50.1 \%)$ & $251(12.7 \%)^{\mathrm{d}}$ & $<0.001^{*}$ \\
\hline $4-8$ & $921(34.2 \%)^{\mathrm{d}}$ & $169(23.6 \%)$ & $752(38.0 \%)^{\mathrm{d}}$ & $<0.001^{*}$ \\
\hline $9-15$ & $1005(37.3 \%)^{\mathrm{d}}$ & $125(17.5 \%)$ & $880(44.5 \%)^{\mathrm{d}}$ & $<0.001^{*}$ \\
\hline $16+$ & $141(5.2 \%)^{\mathrm{d}}$ & $63(8.8 \%)$ & $78(3.9 \%)^{\mathrm{d}}$ & $<0.001^{*}$ \\
\hline \multicolumn{5}{|l|}{ Severity of TBI } \\
\hline Mild & - & $550(76.9 \%)$ & - & - \\
\hline Moderate & - & $65(9.1 \%)$ & - & - \\
\hline Severe & - & $100(14.0 \%)$ & - & - \\
\hline \multicolumn{5}{|l|}{ Comorbidity } \\
\hline \multicolumn{5}{|l|}{$n$ pre-existing conditions } \\
\hline 0 & $1061(39.4 \%)$ & $306(42.8 \%)$ & $755(38.2 \%)$ & $0.032 *$ \\
\hline 1 & $724(26.9 \%)$ & $194(27.1 \%)$ & $530(26.8 \%)$ & 0.861 \\
\hline 2 & $441(16.4 \%)$ & $111(15.5 \%)$ & $330(16.7 \%)$ & 0.473 \\
\hline $3+$ & $467(17.4 \%)$ & $104(14.5 \%)$ & $363(18.5 \%)$ & $0.016^{*}$ \\
\hline \multicolumn{5}{|l|}{$S D$ standard deviation } \\
\hline \multicolumn{5}{|l|}{$*$ Significant at $5 \%$ level } \\
\hline \multicolumn{5}{|l|}{ a 80 missing values } \\
\hline \multicolumn{5}{|l|}{${ }^{\mathrm{b}} 26$ missing values } \\
\hline \multicolumn{5}{|l|}{ c54 missing values } \\
\hline${ }^{\mathrm{d}} 17$ missing values & & & & \\
\hline
\end{tabular}

Table 2 Spearman's rank correlation sum score EQ-5D and EQ-VAS and sum score EQ-5D + C and EQ-VAS for TBI and non-TBI patients

\begin{tabular}{lll}
\hline & Combination & $\begin{array}{l}\text { Spearman's } \\
\text { rank correla- } \\
\text { tion }\end{array}$ \\
\hline Research population & EQ-5D - EQ-VAS & -0.673 \\
& EQ-5D + C - EQ-VAS & -0.690 \\
TBI & EQ-5D - EQ-VAS & -0.719 \\
& EQ-5D + C - EQ-VAS & -0.736 \\
Non-TBI & EQ-5D - EQ-VAS & -0.652 \\
& EQ-5D + C - EQ-VAS & -0.665 \\
\hline
\end{tabular}

$20.4 \%$ ). The explained variance of the EQ-VAS by the cognition dimension was higher in the TBI group than in the non-TBI group for level 2 , as expected, but lower for level 3 , which was unexpected $(8.0 \%$ for level 2 and $9.9 \%$ for level 3 vs. $4.5 \%$ for level 2 and $12.0 \%$ for level 3 ).

The results of the multivariable regression analyses of the group of TBI patients and non-TBI patients are displayed in Table 3 . Generally, $55 \%$ of the variance of the
EQ-VAS could be explained in TBI patients, and $45 \%$ in non-TBI patients. Adding the cognition dimension to the EQ-5D model resulted in an increase in explanatory power from 52.8 to $56.0 \%$ for the TBI group, and from 45.1 to $46.6 \%$ for the non-TBI group. A mutual comparison of explanatory power of all five different selections of five dimensions from six dimensions resulted in similar explained variances, ranging from 52.8 to $54.6 \%$ in TBI patients and 43.3 to $45.4 \%$ in non-TBI patients (see also Fig. 2). The models with additional explanatory variables, resulting from the backward deletion strategy, did provide little additional explanatory power in all cases, except for the EQ-5D + C for TBI patients, where it provided no extra power. The model for the EQ-5D for TBI patients provided an additional explanatory power of $0.9 \%$ when the variable 'Number of TBIs' was added. For the group of nonTBI patients, an additional explanatory power of $1.1 \%$ was found for the EQ-5D $+C$ when the ISS and the number of comorbidities were added, and an additional explanatory power of $1.5 \%$ in the EQ-5D when the same two variables were added. 
Table 3 Explanatory power of multivariable models for the EQ-VAS that include any combination of the EQ-5D and cognition dimensions for TBI and non-TBI patients

\begin{tabular}{llll}
\hline $\begin{array}{l}\text { Combination of EQ-5D +C } \\
\text { dimensions }\end{array}$ & Adjusted $R^{2}$ & $F$ value & $P$ value \\
\hline Research population & & & \\
MO, SC, UA, PD, AD & 0.480 & 249.1 & $<0.001^{*}$ \\
MO, SC, UA, PD, AD, CO & 0.499 & 224.5 & $<0.001^{*}$ \\
MO, UA, PD, AD, CO & 0.486 & 255.4 & $<0.001^{*}$ \\
MO, SC, PD, AD, CO & 0.477 & 246.8 & $<0.001^{*}$ \\
MO, SC, UA, AD, CO & 0.472 & 241.7 & $<0.001^{*}$ \\
MO, SC, UA PD, CO & 0.471 & 241.2 & $<0.001^{*}$ \\
SC, UA, PD, AD, CO & 0.487 & 256.4 & $<0.001^{*}$ \\
TBI patients & & & \\
MO, SC, UA, PD, AD & 0.528 & 80.9 & $<0.001^{*}$ \\
MO, SC, UA, PD, AD, CO & 0.560 & 76.6 & $<0.001^{*}$ \\
MO, UA, PD, AD, CO & 0.546 & 86.7 & $<0.001^{*}$ \\
MO, SC, PD, AD, CO & 0.533 & 82.3 & $<0.001^{*}$ \\
MO, SC, UA, AD, CO & 0.530 & 81.5 & $<0.001^{*}$ \\
MO, SC, UA PD, CO & 0.546 & 86.8 & $<0.001^{*}$ \\
SC, UA, PD, AD, CO & 0.546 & 87.0 & $<0.001^{*}$ \\
Non-TBI patients & & & \\
MO, SC, UA, PD, AD & 0.451 & 163.7 & $<0.001^{*}$ \\
MO, SC, UA, PD, AD, CO & 0.466 & 145.0 & $<0.001^{*}$ \\
MO, UA, PD, AD, CO & 0.452 & 164.3 & $<0.001^{*}$ \\
MO, SC, PD, AD, CO & 0.447 & 161.1 & $<0.001^{*}$ \\
MO, SC, UA, AD, CO & 0.441 & 157.2 & $<0.001^{*}$ \\
MO, SC, UA PD, CO & 0.433 & 151.9 & $<0.001^{*}$ \\
SC, UA, PD, AD, CO & 0.454 & 165.7 & $<0.001^{*}$ \\
\hline
\end{tabular}

$M O$ mobility, $S C$ self-care, $U A$ usual activities, $P D$ pain/discomfort, $A D$ anxiety/depression, $C O$ cognition

*Significant at $5 \%$ level

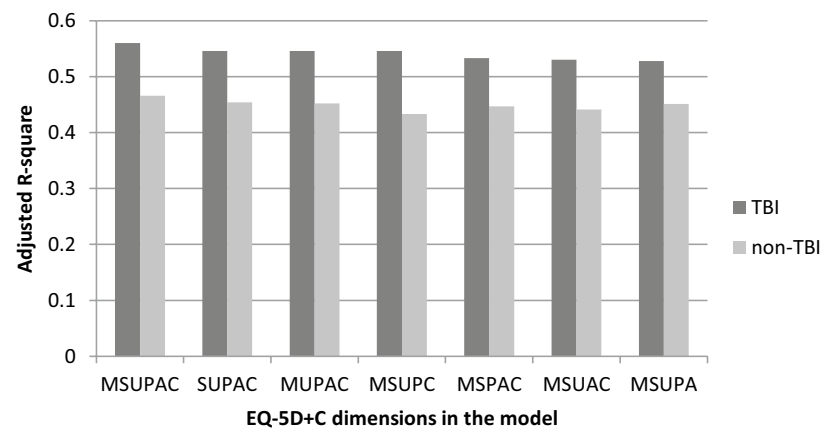

Fig. 2 Adjusted $R^{2}$ per EQ-5D dimensions included for TBI and nonTBI patients for the EQ-VAS. $M$ mobility, $S$ self-care, $U$ usual activities, $P$ pain/discomfort, $A$ anxiety/depression, $C$ cognition

\section{Classification efficiency-Shannon's indices}

The number of different health profiles in the TBI group was considerably lower compared to the non-TBI group.
Table 4 Shannon index $\left(\mathrm{H}^{\prime}\right)$ and Shannon Evenness index $\left(\mathrm{J}^{\prime}\right)$ of EQ-5D and EQ-5D + C for TBI and non-TBI patients

\begin{tabular}{lllll}
\hline Health profiles & $\mathrm{H}^{\prime}$ & Adjusted $\mathrm{H}^{\prime}$ & $\mathrm{J}^{\prime}$ & Adjusted $\mathrm{J}^{\prime}$ \\
\hline TBI & & & & \\
$\quad$ EQ-5D & 5.08 & 5.25 & 0.64 & 0.66 \\
EQ-5D +C & 5.88 & 6.39 & 0.62 & 0.67 \\
Non-TBI & & & & \\
EQ-5D & 5.58 & 5.64 & 0.70 & 0.71 \\
EQ-5D +C & 6.38 & 6.56 & 0.67 & 0.69 \\
\hline
\end{tabular}

In the group of TBI patients, 87 out of 243 (35.8\%) possible EQ-5D profiles were used, and 153 out of $729(21.0 \%)$ possible EQ-5D $+\mathrm{C}$ profiles were reported. In the group of non-TBI patients, 127 out of $243(52.3 \%)$ possible EQ-5D profiles and 232 out of $729(31.8 \%)$ possible EQ-5D +C profiles were reported. This was reflected in the Shannon index and the Shannon Evenness index, which were 5.08 (Shannon index) and 0.64 (Shannon Evenness index) for the EQ-5D and 5.88 (Shannon index) and 0.62 (Shannon Evenness index) for the EQ-5D $+\mathrm{C}$, respectively, for the TBI group, while for the non-TBI group the results were 5.58 and 0.70 for the EQ-5D, and 6.38 and 0.67 for the EQ-5D + C (Table 4). Apart from the non-TBI group being more heterogeneous, this indicates that generally more information is captured by the EQ-5D $+C$ compared to the EQ-5D. The Shannon Evenness index was higher for the EQ-5D than for the EQ-5D $+\mathrm{C}\left(J^{\prime}=0.64\right.$ vs. $J^{\prime}=0.62$ for TBI, and $J^{\prime}=0.70$ vs. $J^{\prime}=0.67$ for non-TBI), but were similar after adjusting for underestimation bias $\left(J_{\text {adj }}^{\prime}=0.66\right.$ vs. $J^{\prime}{ }_{\text {adj }}=0.67$ for TBI, and $J^{\prime}{ }_{\text {adj }}=0.71$ vs. $J_{\text {adj }}^{\prime}=0.69$ for non-TBI). Apparently, more information is captured but the observed gain in discrimination in both groups is relatively low in view of the increase of classification options (adding cognition increases the options with $729-243=585$ profiles).

\section{Discussion}

\section{Main findings}

This study addressed the potential gain of adding a cognitive dimension to the EQ-5D measure, in a large group of injury patients where this gain could be relevant in the subgroup of TBI patients. Genuinely it slightly improved ceiling effects and improved the ranking of patients if a self-rated VAS was used as reference. As expected, this effect was larger in TBI patients. However, the increase in explanatory power was rather small (3.2\% if we focus on TBI). Furthermore, the added value in terms of explanatory power of the cognition dimension in the non-TBI population was close to zero, 
as expected. Discriminatory potential (informativity) was higher for the EQ-5D + C than for the EQ-5D in both groups, whereas relative informativity was similar.

\section{Comparison to previous studies}

The addition of a cognition dimension to the EQ-5D has been studied before, but not specifically in a population with TBI. Ophuis et al. (working paper) looked into the effect in a small group of TBI patients, but a clinical classification of severity of the TBI was lacking. Moreover, in the study by Ophuis et al. (working paper) HRQoL was assessed 2.5 months after ED treatment, whereas in this study the EQ-5D $(+C)$ was assessed 1 month after ED treatment, and as a result we expect that less patients fully recovered from their injuries. Despite these differences, the findings of the study by Ophuis et al. (working paper) were similar to our findings: compared to EQ-5D the increase in explanatory power of the EQ-5D $+\mathrm{C}$ is small $(<3 \%)$.

Findings of other studies on the cognition dimension are more difficult to compare. Krabbe et al. [10] looked into the effect of adding a cognition dimension to the EQ-5D; however, in this study the content validity was tested, as well as the impact on utilities. The study by Jelsma and Maart [7] looked into the effect of adding several dimensions, including a concentration dimension, to the EQ-5D. Concentration is considered a part of the cognition dimension. The study by Jelsma and Maart [7] found that the addition of a concentration dimension increased the explanatory power of the model (52-55\% when concentration, energy, body appearance, sleep, and sexual activity were added). However, they did not report the increase in explanatory value for each extra dimension separately.

Convergent validity of the EQ-5D has been studied by Golicki et al. [20]. In this study, the convergent validity was determined for each dimension of the EQ-5D with the EQ-VAS, with the Spearman rank correlation coefficients ranging from -0.43 for pain/discomfort to -0.75 for usual activities [20]. These correlation coefficients are in the same order of magnitude compared to those we found for the EQ-5D and EQ-5D + C.

Previous studies on the classification efficiency of the EQ-5D found varying values for the Shannon index and the Shannon Evenness index of the EQ-5D. Janssen, Birnie, and Bonsel [21] found a Shannon index of 6.37 and a Shannon Evenness index of 0.80 in a general population sample from the US. Polinder et al. [22] reported a Shannon index for the EQ-5D of 2.71 and a Shannon Evenness index of 0.53 in an injury population. For skull and brain injury patients, this study reported a Shannon index of 2.55 for the EQ-5D and a Shannon Evenness index of 0.49 [22]. The Shannon index that was found in our injury population is higher ( 5.08 for TBI and 5.58 for non-TBI). This can be explained by the fact that the measurement of HRQoL in our study was conducted 1 month after presentation at the ED, versus 2 years after injury. Therefore, it is likely that there was a larger variation of health profiles in our study than in the study by Polinder et al. [22], resulting in a higher Shannon index and Shannon Evenness index. It should be taken into account that the ceiling effect is also part of the formula to determine the classification efficiency. However, in calculating the classification efficiency it is not relevant whether a high frequency of one health profile is in the perfect health profile (11111) or in another health profile. Therefore, if a health profile is overrepresented, this will result in a smaller classification efficiency.

\section{Strengths and limitations}

This study had several strengths and limitations. One of the strengths was that diagnosis and severity of TBI was registered. A limitation of this study was the timing of the HRQoL assessment. A study by Schretlen and Shapiro [23] found that cognitive functioning after mild TBI restores within 1-3 months. Since the majority of patients in the TBI group had mild TBI, patients may have already recovered 1 month after presentation at the ED. The BIOS study also contains data on a 1-week measurement; however, the number of respondents at this point is rather small. Secondly, the group of non-TBI patients had on average a significantly higher ISS score, indicating that this group of patients sustained more severe injuries compared to the group of TBI patients. This is reflected by the much higher proportion of patients in the TBI group who reported perfect health. The EQ-5D was found to suffer from ceiling effect, meaning that health states close to good health cannot be distinguished well $[18,24]$. Therefore, the ceiling effect was larger in the TBI group than in the non-TBI group. A question that arises is whether the ceiling effect in the TBI group (18.0\% for EQ-5D and $16.4 \%$ for EQ-5D +C) would have been reduced when the EQ-5D-5L or EQ-5D-5L + C was used, as is suggested by Janssen et al. [18]. Respondents with minor complaints on one of the dimensions will choose 'no problems' in the EQ-5D-3L, but are likely to choose 'slight problems' in the EQ-5D-5L.

For future research, we recommend to assess the added value of the cognitive bolt-on for the EQ-5D-5L and to include more patients with moderate or severe TBI to enable a comparison between the severity levels of TBI. Furthermore, we recommend to develop guidelines to determine what a meaningful difference, in a statistical sense, of a bolton is. 


\section{Conclusion}

In conclusion, the addition of a cognition dimension to the EQ-5D improves the convergent validity, explanatory power, and classification efficiency of the EQ-5D. However, improvements are rather small. The effect on the convergent validity and the explanatory power of the EQ-5D is larger in the group of TBI patients than in the group of non-TBI patients, suggesting that the additional value of a cognition dimension in the EQ-5D is especially relevant in a patient group with (expected) problems with cognition.

\section{Compliance with ethical standards}

Conflict of interest All the authors declare that they have no conflict of interest.
Ethical approval All procedures performed involving human participants were in accordance with the 1964 Helsinki declaration and its later amendments or comparable ethical standards, and the study was approved by the Medical Ethics Committee of the Province of Brabant (METC code: NL50258.028.14).

Informed consent Informed consent was obtained from all individual participants included in the study.

Open Access This article is distributed under the terms of the Creative Commons Attribution 4.0 International License (http://creativeco mmons.org/licenses/by/4.0/), which permits unrestricted use, distribution, and reproduction in any medium, provided you give appropriate credit to the original author(s) and the source, provide a link to the Creative Commons license, and indicate if changes were made.

\section{Appendix}

Table 5 Univariate analyses of EQ-VAS explained by the EQ-5D and cognition dimensions for TBI and nonTBI patients

\begin{tabular}{|c|c|c|c|}
\hline EQ-5D dimension & $R^{2}$ & Unstandardized B $(95 \% \mathrm{CI})$ & $p$ value \\
\hline \multicolumn{4}{|l|}{ TBI patients } \\
\hline Mobility level 2 & 0.116 & $-13.4(-16.1$ to -10.7$)$ & $<0.001 *$ \\
\hline Mobility level 3 & 0.109 & $-33.3(-40.3$ to -26.3$)$ & $<0.001 *$ \\
\hline Self-care level 2 & 0.076 & $-12.1(-15.3$ to -9.0$)$ & $<0.001 *$ \\
\hline Self-care level 3 & 0.156 & $-28.3(-33.2$ to -23.5$)$ & $<0.001 *$ \\
\hline Usual activities level 2 & 0.009 & $-3.7(-6.6$ to -0.9$)$ & $0.005^{*}$ \\
\hline Usual activities level 3 & 0.243 & $-24.2(-27.3$ to -21.0$)$ & $<0.001 *$ \\
\hline Pain/discomfort level 2 & 0.038 & $-7.8(-10.8$ to -4.9$)$ & $<0.001 *$ \\
\hline Pain/discomfort level 3 & 0.131 & $-26.3(-31.3$ to 21.3$)$ & $<0.001 *$ \\
\hline Anxiety/depression level 2 & 0.123 & $-15.4(-18.4$ to -12.4$)$ & $<0.001 *$ \\
\hline Anxiety/depression level 3 & 0.062 & $-25.5(-32.8$ to -18.2$)$ & $<0.001 *$ \\
\hline Cognition level 2 & 0.080 & $-11.7(-14.5$ to -8.8$)$ & $<0.001 *$ \\
\hline Cognition level 3 & 0.099 & $-29.7(-36.3$ to -23.1$)$ & $<0.001 *$ \\
\hline \multicolumn{4}{|l|}{ Non-TBI patients } \\
\hline Mobility level 2 & 0.005 & $-2.8(-4.6$ to -1.1$)$ & $0.002 *$ \\
\hline Mobility level 3 & 0.114 & $-20.1(-22.6$ to -17.7$)$ & $<0.001 *$ \\
\hline Self-care level 2 & 0.002 & $-1.8(-3.5$ to -0.1$)$ & $0.043^{*}$ \\
\hline Self-care level 3 & 0.204 & $-23.6(-25.6$ to -21.5$)$ & $<0.001 *$ \\
\hline Usual activities level 2 & 0.050 & $8.8(7.1$ to 10.5$)$ & $<0.001 *$ \\
\hline Usual activities level 3 & 0.204 & $-18.0(-19.6$ to -16.5$)$ & $<0.001 *$ \\
\hline Pain/discomfort level 2 & 0.003 & $-2.4(-4.3$ to -0.5$)$ & $0.014^{*}$ \\
\hline Pain/discomfort level 3 & 0.088 & $-19.8(-22.6$ to -17.0$)$ & $<0.001 *$ \\
\hline Anxiety/depression level 2 & 0.095 & $-13.3(-15.1$ to -11.5$)$ & $<0.001 *$ \\
\hline Anxiety/depression level 3 & 0.102 & $-28.4(-32.1$ to -24.7$)$ & $<0.001 *$ \\
\hline Cognition level 2 & 0.045 & $-9.9(-11.9$ to -7.9$)$ & $<0.001 *$ \\
\hline Cognition level 3 & 0.120 & $-26.5(-29.7$ to -23.4$)$ & $<0.001 *$ \\
\hline
\end{tabular}

95\% $C I=95 \%$ confidence interval

*Significant at $5 \%$ level 


\section{References}

1. Gabbe, B. J., Simpson, P. M., Cameron, P. A., Ponsford, J., Lyons, R. A., Collie, A., et al. (2017). Long-term health status and trajectories of seriously injured patients: A population-based longitudinal study. PLoS Medicine, 14(7), e1002322.

2. Patrick, D. L., Deyo, R. A. (1989). Generic and disease-specific measures in assessing health status and quality of life. Medical Care, 27(3), S217-S232.

3. Brooks, R. (1996). EuroQol: The current state of play. Health Policy, 37(1), 53-72.

4. Linde, L., Sorensen, J., Ostergaard, M., Horslev-Petersen, K., \& Hetland, M. L. (2008). Health-related quality of life: Validity, reliability, and responsiveness of SF-36, 15D, EQ-5D [corrected] RAQoL, and HAQ in patients with rheumatoid arthritis. Journal of Rheumatology, 35(8), 1528-1537.

5. Johnson, J. A., Coons, S. J., Ergo, A., \& Szava-Kovats, G. (1998). Valuation of EuroQOL (EQ-5D) health states in an adult US sample. Pharmacoeconomics, 13(4), 421-433.

6. Lin, F. J., Longworth, L., \& Pickard, A. S. (2013). Evaluation of content on EQ-5D as compared to disease-specific utility measures. Quality of Life Research, 22(4), 853-874.

7. Jelsma, J., \& Maart, S. (2015). Should additional domains be added to the EQ-5D health-related quality of life instrument for community-based studies? An analytical descriptive study. Population Health Metrics, 13, 13.

8. Finch, A. P., Brazier, J. E., \& Mukuria, C. (2018). What is the evidence for the performance of generic preference-based measures? A systematic overview of reviews. The European Journal of Health Economics, 19(4), 557-570.

9. Yang, Y., Rowen, D., Brazier, J., Tsuchiya, A., Young, T., \& Longworth, L. (2015). An exploratory study to test the impact on three "bolt-on" items to the EQ-5D. Value Health, 18(1), 52-60.

10. Krabbe, P. F., Stouthard, M. E., Essink-Bot, M. L., \& Bonsel, G. J. (1999). The effect of adding a cognitive dimension to the EuroQol multiattribute health-status classification system. Journal of Clinical Epidemiology, 52(4), 293-301.

11. Wolfs, C. A., Dirksen, C. D., Kessels, A., Willems, D. C., Verhey, F. R., \& Severens, J. L. (2007). Performance of the EQ-5D and the EQ-5D $+\mathrm{C}$ in elderly patients with cognitive impairments. Health Qual Life Outcomes, 5, 33.

12. de Jongh, M. A., Kruithof, N., Gosens, T., van de Ree, C. L., de Munter, L., Brouwers, L., et al. (2017). Prevalence, recovery patterns and predictors of quality of life and costs after non-fatal injury: The Brabant Injury Outcome Surveillance (BIOS) study. Injury Prevention, 23(1), 59.
13. Baker, S. P., O’Neill, B., Haddon, W. Jr., \& Long, W. B. (1974). The injury severity score: A method for describing patients with multiple injuries and evaluating emergency care. The Journal of Trauma, 14(3), 187-196.

14. Gennarelli, T. A., \& Wodzin, E. (2006). AIS 2005: A contemporary injury scale. Injury, 37(12), 1083-1091.

15. van der Molen, T., Willemse, B. W., Schokker, S., ten Hacken, N. H., Postma, D. S., \& Juniper, E. F. (2003). Development, validity and responsiveness of the Clinical COPD Questionnaire. Health and Quality of Life Outcomes, 1, 13.

16. Shannon, C. E. (1997). The mathematical theory of communication 1963. MD Computing, 14(4), 306-317.

17. Pickard, A. S., De Leon, M. C., Kohlmann, T., Cella, D., \& Rosenbloom, S. (2007). Psychometric comparison of the standard EQ-5D to a 5 level version in cancer patients. Medical Care, 45(3), 259-263.

18. Janssen, M. F., Pickard, A. S., Golicki, D., Gudex, C., Niewada, M., et al. (2013). Measurement properties of the EQ-5D-5L compared to the EQ-5D-3L across eight patient groups: A multicountry study. Quality of Life Research, 22(7), 1717-1727.

19. Pielou, E. C. (1966). Shannon's Formula as a measure of specific diversity: Its use and misuse. The American Naturalist, 100(914), 463-465.

20. Golicki, D., Niewada, M., Buczek, J., Karlinska, A., Kobayashi, A., Janssen, M. F., et al. (2015). Validity of EQ-5D-5L in stroke. Quality of Life Research, 24(4), 845-850.

21. Bas Janssen, M. F., Birnie, E., \& Bonsel, G. J. (2007). Evaluating the discriminatory power of EQ-5D, HUI2 and HUI3 in a US general population survey using Shannon's indices. Quality of Life Research, 16(5), 895-904.

22. Polinder, S., Haagsma, J. A., Bonsel, G., Essink-Bot, M. L., Toet, H., \& van Beeck, E. F. (2010). The measurement of long-term health-related quality of life after injury: Comparison of EQ-5D and the health utilities index. Injury Prevention, 16(3), 147-153.

23. Schretlen, D. J., \& Shapiro, A. M. (2003). A quantitative review of the effects of traumatic brain injury on cognitive functioning. International Review of Psychiatry, 15(4), 341-349.

24. Brazier, J., Roberts, J., Tsuchiya, A., \& Busschbach, J. (2004). A comparison of the EQ-5D and SF-6D across seven patient groups. Health Economics, 13(9), 873-884.

Publisher's Note Springer Nature remains neutral with regard to jurisdictional claims in published maps and institutional affiliations. 